\title{
Incorporating patient preference into the management of infertility in women with polycystic ovary syndrome
}

This article was published in the following Dove Press journal:

Patient Preference and Adherence

23 May 2012

Number of times this article has been viewed

\author{
Ugochi C Okoroafor \\ Emily $S$ Jungheim \\ Department of Obstetrics and \\ Gynecology, Washington University, \\ St Louis, MO, USA
}

\begin{abstract}
Polycystic ovary syndrome (PCOS) is a heterogeneous condition characterized by anovulation, hyperandrogenism, and polycystic ovaries. Because of the heterogeneous nature of PCOS, women affected by the condition often require a customized approach for ovulation induction when trying to conceive. Treating symptoms of PCOS in overweight and obese women should always incorporate lifestyle changes with the goal of weight-loss, as many women with PCOS will ovulate after losing 5\%-10\% of their body weight. On the other hand, other factors must be considered including the woman's age, age-related decline in fertility, and previous treatments she may have already tried. Fortunately, multiple options for ovulation induction exist for women with PCOS. This paper reviews specific ovulation induction options available for women with PCOS, the benefits and efficacy of these options, and the related side effects and risks women can anticipate with the various options that may affect treatment adherence. The paper also reviews the recommended evidence-based strategies for treating PCOS-related infertility that allow for incorporation of the patient's preference. Finally, it briefly reviews emerging data and ongoing studies regarding newer agents that have shown great promise as first-line agents for the treatment of infertility in women with PCOS.
\end{abstract}

Keywords: polycystic ovary syndrome, anovulation, clomiphene citrate, letrozole, metformin, obesity

\section{Treating PCOS-related infertility in the 2 I st century}

Stein and Leventhal initially described polycystic ovarian syndrome (PCOS) with associated amenorrhea in $1935 .{ }^{1}$ They also described a strategy called an ovarian wedge resection that seemed to be effective in treating the associated infertility. Since that time, numerous descriptions and diagnostic criterion for the condition have emerged. While it is fortunate that other treatment strategies have emerged, because varying diagnostic criteria have been used to define PCOS over the years, it can be difficult to determine the external validity of older published PCOS literature. More recently, increasing attention to the exact definitions of PCOS and the quality of clinical studies investigating treatments for infertility related to PCOS have greatly advanced our understanding of the nuances involved in treating PCOS-related infertility. The following sections review the evidence for the efficacy of various infertility treatment strategies as well as the side effects and risks that may affect patient adherence. Overall, this review examines the clinical experience and evidence for treating PCOS-related infertility so that a treatment plan can be constructed that incorporates patient preference and is mutually satisfactory for the patient and the clinician.
Correspondence: Emily S Jungheim Division of Reproductive Endocrinology and Infertility, Department of Obstetrics and Gynecology, Washington University in St Louis, St Louis, MO 63108, USA $\mathrm{Tel}+\mathrm{I} 3 \mid 42862400$

Fax + I 3142862455

Email jungheime@wudosis.wustl.edu submit your manuscript | www.dovepress.com

Dovepress

http://dx.doi.org// 0.2147/PPA.S25286 


\section{A current working definition of PCOS}

As previously mentioned, PCOS was originally described by Stein and Leventhal in their classic paper published in the American Journal of Obstetrics and Gynecology in 1935. ${ }^{1}$ In this paper, Stein and Leventhal described seven women with enlarged, polycystic ovaries, obesity, hirsutism, and chronic anovulation. Recognizing the need to establish diagnostic criteria for PCOS to allow for well-designed research and clinical trials related to PCOS, an expert panel sponsored by the National Institutes of Health (NIH) developed a widely accepted definition for PCOS in $1990 .^{2}$ This definition included the following major criteria for defining PCOS: hyperandrogenism and/or hyperandrogenemia, menstrual dysfunction, and the exclusion of other known disorders. While these criteria helped to clarify the standards for diagnosis, treatment, and research, debate still existed on the criteria that should be used to define the condition. In 2003, another expert conference was convened in Rotterdam, The Netherlands. This conference was sponsored by the European Society for Human Reproduction and Embryology and the American Society for Reproductive Medicine. At this conference, the experts agreed that PCOS should be defined by the presence of two of the following characteristics: oligo and/or anovulation, clinical and/or biochemical signs of hyperandrogenism, and/or polycystic ovaries on an ultrasound. This definition was broader than the NIH criteria and placed less emphasis on the hyperandrogenism requirement. More recently, the Androgen Excess and PCOS Society, an organization comprising international experts in the area of PCOS, published a report outlining the following characteristics as being key to the diagnosis of PCOS: hyperandrogenism (clinical and/or biochemical), ovarian dysfunction (oligo-anovulation and/or polycystic ovaries), and the exclusion of related disorders. In this definition, the experts re-established hyperandrogenism as the key to PCOS diagnosis.

While some of the debate surrounding the definition of PCOS may be related to semantics for research purposes rather than clinical care, establishing clear diagnostic criteria for PCOS is necessary for embarking on sophisticated mechanistic research involving the study of PCOS and for applying the results of clinical trials to individual patients.

Regardless of the criteria used to diagnose women affected by PCOS, it is the most common endocrinopathy affecting women of reproductive age. Somewhere between $6 \%-8 \%$ of women are affected by this syndrome worldwide. ${ }^{3}$ In addition to the hyperandrogenism and anovulation/oligomenorrhea that characterize the condition, depression, and long-term risks for the development of endometrial cancer, diabetes, and cardiovascular disease are also significant risks. ${ }^{4-6}$ If no work up has been performed on a woman presenting with a clinical picture of PCOS, we will typically screen these women for other causes of ovulation disorders including thyroid disease, Cushing's syndrome, and premature ovarian failure when appropriate. We also typically check a serum testosterone level (total), and a 17-hydroxyprogesterone value in addition to routine screening tests recommended by current guidelines including a complete serum lipid panel and a 2 hour, 75 gram oral glucose tolerance test. ${ }^{6}$

\section{Opportunity for preconception counseling in infertile women with PCOS: an opportunity for education and improved lifestyle for a healthier outcome Screening for glucose intolerance and diabetes - to take metformin or not?}

Current guidelines for treating women with PCOS recommend screening for glucose intolerance. ${ }^{6}$ Of the available options for screening (fasting glucose, hemoglobin $A_{1 c}$, and the 2 hour, 75 gram oral glucose tolerance test), the 2 hour, 75 gram oral glucose tolerance test may be the most sensitive means of detecting glucose intolerance. ${ }^{5,7}$ This method may also be helpful for determining if a woman is a pregestational diabetic. For women who are not diabetic, but who are glucose intolerant, the addition of metformin may lead to improved insulin sensitivity and a better metabolic profile and response to clomiphene citrate (CC) for ovulation induction. ${ }^{8}$ Metformin will likely have no benefit for non-obese women with PCOS who do not show glucose intolerance after screening. Whether or not metformin reduces the risk of developing gestational diabetes once pregnancy occurs is unknown although more recent data suggests that it does not. ${ }^{9}$ Including this background information for women when counseling them on their treatment options for ovulation induction may be helpful as metformin was touted as the mainstay of PCOS treatment by many for years, but unfortunately, many women experience gastrointestinal side effects such as nausea and diarrhea. Due to the risks of associated lactic acidosis, metformin should be started at a low dose and built up over time, and only if women are proven to have normal renal and hepatic functioning. We recommend starting at $500 \mathrm{mg}$ po daily for one week with an additional 
$500 \mathrm{mg}$ daily per week until a $2000 \mathrm{mg}$ daily dose is reached. The extended release formulation may be beneficial for women who have difficulty with that dosage level.

\section{Weight loss}

While infertility is an emotionally difficult condition for any couple to face, it offers an important opportunity for thorough preconception counseling and intervention for couples hoping to conceive. In women with PCOS, this opportunity can be especially beneficial as these women face increased risks of gestational diabetes and preeclampsia during pregnancy. These risks, along with the anovulation associated with PCOS, are often exacerbated by the excess body weight that often accompanies PCOS . Overweight and obese women with anovulation/oligomenorrhea and PCOS will often ovulate spontaneously after losing only $5 \%-10 \%$ of their body weight. When setting a timeline and goal for weight loss, it is important to keep the woman's age in mind as fertility decreases more rapidly after age 35 . Morbidly obese women with a body mass index (BMI) of $40 \mathrm{~kg} / \mathrm{m}^{2}$ or greater may require greater weight loss. ${ }^{10}$ For many women, clinically significant weight loss may be difficult to achieve and sustain. Encouraging partner involvement in the weight loss plan may be helpful. Reviewing previous attempts at weight loss with women and encouraging them to consider commercial weight loss programs may also be beneficial along with a referral to a dietician and an exercise facility. Setting a goal for weight loss and a plan for follow up are also important. This is also an opportune time to review the long-term health risks associated with obesity and PCOS such as type 2 diabetes, endometrial cancer, and cardiovascular disease. Reviewing these risks with women at this crucial time may encourage them to act.

For obese women with PCOS who are over $40 \mathrm{~kg} / \mathrm{m}^{2}$ or women who are $35 \mathrm{~kg} / \mathrm{m}^{2}$ with co-morbidities like diabetes, bariatric surgery may be an option for achieving sustainable weight loss. Again, it may be necessary for these women to lose more than $5 \%-10 \%$ of their body weight to see improvements in ovulation. On the other hand, bariatric surgery is not without risks and requires a commitment that many people are not willing to make. Nevertheless, this option is worth mentioning to women who qualify, along with a discussion of the time commitment required. Most surgeons recommend that women wait for a period of one year after bariatric surgery prior to conception so that the maximum benefit of the weight loss associated with the procedure can be achieved. Many women have successfully conceived prior to the one-year wait period and this may be reasonable for older patients. ${ }^{11}$ It is important to warn patients that while they may achieve successful weight loss, fertility treatments may still be required. ${ }^{12}$

Given the time commitment required for weight loss, a discussion of patient age and baseline fertility is warranted since delaying fertility treatment may be detrimental to a woman's prospects for childbearing. Recent crosssectional studies by the Society for Assisted Reproductive Technologies (SART) present convincing evidence on the impact of obesity on fertility versus the impact of age. In these studies, researchers have shown that increasing obesity is associated with decreased chances of conception in women younger than age 35 undergoing assisted reproductive technologies (ART). ${ }^{13}$ Conversely, in women over the age of 35 , obesity has less of an impact on the success of ART and age becomes the overriding factor. ${ }^{13,14}$ While attempts at weight loss and lifestyle improvements should never be discouraged in overweight and obese women, women must be fully informed of the realistic expectations they may have for fertility with increasing age.

\section{Modifiable risk factors: dietary interventions}

Emerging epidemiologic data from the Nurses' Health Study II (NHSII) suggest that certain foods may be associated with ovulation disorders like PCOS. ${ }^{15}$ While controlling for BMI, nutritional epidemiologists involved with NHSII have demonstrated that certain dietary choices may be associated with higher risks of anovulation. The results of these epidemiologic investigations led by nutritional epidemiologist, Jorge Chavarro, $\mathrm{MD}, \mathrm{PhD}$, suggest that saturated animal fats and trans-fats found in commercially baked goods and some fried fast foods are associated with ovulatory infertility while fats found in foods such as salmon, tuna, and nut sources may be beneficial to promoting ovulatory function. ${ }^{16}$ Dr Chavarro's work also shows that foods containing refined sugars are detrimental to ovulatory fertility while carbohydrate-rich foods with a lower glycemic index may be beneficial. ${ }^{17}$ Finally, these studies have shown that daily multi-vitamin use may be beneficial for women with PCOS-related infertility along with supplemental iron intake. ${ }^{18,19}$ Given that folic acid is an important supplement for any woman of reproductive age for preventing neural tube defects, a multi-vitamin containing folic acid should always be encouraged. Education regarding patient-modified interventions such as these may be empowering for some patients and should be 
discussed along with the disclaimer that prospective trials incorporating dietary recommendations like these have yet to be completed; therefore, their impact on ovulation and reproductive outcomes is yet to be determined.

\section{When time runs out: moving on from lifestyle intervention to ovulation induction}

As mentioned, fertility declines with age. This decline increases rapidly after age 35 . It has been suggested in the SART literature that women younger than 35 aim for a BMI of less than $30 \mathrm{~kg} / \mathrm{m}^{2} .{ }^{20}$ Women over age 35 should aim for a BMI of less than $35 \mathrm{~kg} / \mathrm{m}^{2}$. For these women, it is unknown whether achieving a BMI of less than $35 \mathrm{~kg} / \mathrm{m}^{2}$ will lead to an improved response to fertility treatment or a healthier pregnancy outcome. It is both realistic and reasonable to propose fertility treatment for otherwise healthy overweight and obese women with PCOS who have tried to lose weight unsuccessfully, and this approach is supported by the Ethics Committee of the American Society for Reproductive Medicine. ${ }^{21}$ While obesity is associated with decreased success in fertility treatments ${ }^{22}$ and increased risks of adverse pregnancy outcomes, a BMI at which it is unsafe to provide fertility treatments has not been established and likely never will be since there are numerous examples of women with excessive BMIs who have successfully conceived and delivered. Ultimately, a conversation between the physician and patient must take place to determine the right course of action and to understand the evidence regarding the competing risks of time required for weight loss and the effects of age on fertility and response to fertility treatment.

\section{The data for ovulation induction in women with PCOS: past, present and future Treatments of the past: wedge resection and ovarian drilling}

As mentioned in the introduction, Stein and Leventhal described wedge resection for ovulation induction in women with PCOS. ${ }^{1}$ While this method can help women achieve spontaneous ovulation, it is invasive with significant risk of blood loss during surgery, post-operative adhesion formation, and time-loss for recovery. Ovarian drilling has also been shown to be efficacious in inducing spontaneous ovulation and pregnancy. ${ }^{23-25}$ It offers an advantage over ovarian wedge resection as it is less likely to be associated with significant blood loss, and is easily conducted via a laparoscopic technique. This method is helpful in cases where women are resistant to ovulation induction with oral medications and want to avoid gonadotropin injections. However, it is also associated with pelvic adhesion formation and decreased ovarian reserve over time as a portion of the ovary is destroyed through the drilling process. Both of these surgical interventions have been largely replaced with oral ovulation induction methods with gonadotropin injections, while ARTs are reserved for women who fail to ovulate or conceive with oral medications.

\section{First-line treatment: CC, metformin, or both?}

$\mathrm{CC}$ is a convenient, accessible, and relatively inexpensive treatment that has been a mainstay for ovulation induction in women with PCOS for over 40 years. Unfortunately, a fraction of women with PCOS are resistant to CC. In the 1990s, metformin was identified as a solution to such cases and gained popularity as a first-line alternative to $\mathrm{CC}$ because it was thought to improve metabolic functioning while decreasing the risk PCOS in patients who faced developing gestational diabetes during pregnancy. ${ }^{8,9,26}$ Numerous studies have emerged that compare the two agents for ovulation induction with disparate results. ${ }^{27,28}$ Finally, the NIH-sponsored Reproductive Medicine Network (NIH-RMN) undertook a large, multi-centered trial comparing $\mathrm{CC}$, metformin, and both as agents for ovulation induction. ${ }^{29}$ In this intention-to-treat trial, CC was clearly better as a first-line agent for achieving pregnancy and live birth when compared to metformin alone. The combination of CC with metformin was equivocal, although there was some evidence that the combination arm may have helped in PCOS patients with morbid obesity. Some have argued that it was poor compliance with metformin therapy that resulted in the superior results of the $\mathrm{CC}$-alone arm, ${ }^{30}$ but a secondary analysis of the data has demonstrated that adherence in both the metformin-only and combinedmetformin-CC arms was within the reported expectations for clinical trials. ${ }^{31}$ The authors of the study concluded that failure to comply with metformin dosing was not the reason for low ovulation and pregnancy rates in the metformin arms of the trial.

Common side-effects associated with $\mathrm{CC}$ include hot flashes and labile mood. Visual disturbances and headaches should warrant discontinuation and consideration of switching to an alternative agent for ovulation induction. We recommend starting with a daily dose of CC $50 \mathrm{mg}$ daily cycle for 3-7 day with a luteal phase progesterone level to document ovulation. If the progesterone value is $<2 \mathrm{ng} / \mathrm{mL}$ 
and the woman's cycle is longer than 35 days with a negative urine pregnancy test, we recommend inducing menses and proceeding with another cycle of CC and increasing the dose by an additional $50 \mathrm{mg}$ cycle for 3-7 days. These steps are to be repeated until ovulation is achieved to a maximum dose of $150 \mathrm{mg}$ to $200 \mathrm{mg}$ depending on the associated side effects. This process can be frustrating and it is often important to encourage women to be persistent if ovulation is not achieved in the first one or two cycles to help with adherence. ${ }^{31}$

For women who are resistant to ovulation induction with $\mathrm{CC}$ alone, the addition of metformin may be beneficial. ${ }^{26}$ In a recent publication, we demonstrated that initial concomitant treatment with $\mathrm{CC}$ and metformin may be superior to CC alone. ${ }^{32}$ We argue that the sample size employed in the NIH-RMN trial was not large enough to detect the clinically significant difference that may exist in treating women with $\mathrm{CC}$ alone versus $\mathrm{CC}$ and metformin together. ${ }^{33}$ Again, screening for insulin resistance in women with PCOS prior to treatment may indicate which women will benefit from initial concomitant treatment with both $\mathrm{CC}$ and metformin.

As previously discussed, metformin - labeled by some as "Vitamin M" - was the first-line for treating infertility in women with PCOS. ${ }^{34}$ Due to the heterogeneous nature of the PCOS, not all women will respond to metformin; therefore, it should be reserved for women with proven insulin-resistance. Again, we recommend a 2 hour, 75 gram oral glucose tolerance test for screening. We also recommend that it not be used alone in the treatment of PCOS-related infertility as the results of the NIH-RMN trial described above clearly demonstrate the inadequacy of metformin alone when compared to $\mathrm{CC}$ alone and $\mathrm{CC}$ and metformin together. For women who are insulin-resistant, we recommend an approach of $\mathrm{CC}$ at $50 \mathrm{mg}$ daily cycle for 3-7 days with a daily dose of $2000 \mathrm{mg}$ of metformin. As outlined above, metformin should be started at a daily dose of $500 \mathrm{mg}$ and built up over a period of weeks to reach the $2000 \mathrm{mg}$ dose.

\section{Letrozole as the future first-line treatment}

Aromatase inhibitors prevent the aromatization of testosterone to estrogen and are used primarily as adjunctive agents to treat breast cancer. The first report of letrozole's efficacy at inducing ovulation in women with PCOS emerged in $2001 .{ }^{35}$ Letrozole is relatively well tolerated in terms of the associated side effects such as gastrointestinal disturbances and hot flashes. Furthermore, letrozole has a shorter half-life than CC. When used for ovulation induction, letrozole is associated with a thicker endometrium compared to $\mathrm{CC}$ and multiple gestations may be less common. While letrozole appears to be efficacious at inducing ovulation, it is yet to be demonstrated in adequately designed clinical trials whether it results in higher pregnancy rates and an improved chance at singleton pregnancy compared to CC. ${ }^{36-38}$ Fortunately, the RMN has initiated a large, multi-centered trial to investigate the effect of letrozole versus $\mathrm{CC}$ in infertile women with PCOS. ${ }^{39}$ The primary outcome of this trial is live birth. Important secondary outcomes include, among others, singleton pregnancy, incidence of first trimester fetal demise, time to pregnancy, quality of life while on treatment, and cost-effectiveness.

Letrozole is currently reserved for women with PCOSrelated infertility who do not respond to ovulation induction with $\mathrm{CC}$ or who cannot tolerate its side effects. We typically prescribe $5 \mathrm{mg}$ of letrozole for a 3-7 day cycle with a midluteal serum progesterone level to document ovulation.

If ovulation is not achieved with lifestyle interventions or oral agents or a woman fails to conceive in a given time frame, we recommend moving on to a more aggressive treatment. We do not recommend treating women with ovarian wedge resection or laparoscopic ovarian drilling. We will typically treat a woman with ovulation induction agents for up to six ovulatory cycles provided that tubal patency and a reasonable semen analysis have been noted. In general, if a woman with PCOS is not pregnant after six ovulatory cycles and she is ready to move on to other treatment options, we will do so.

\section{When all else fails: controlled ovarian hyperstimulation and in vitro fertilization Controlled ovarian hyperstimulation with injectable medications}

We reserve controlled ovarian hyperstimulation $(\mathrm{COH})$ for women with PCOS who have failed ovulation induction with oral agents. Our goal in treating PCOS patients with $\mathrm{COH}$ is to achieve monofollicular development. To achieve this goal, a lower starting dose of gonadotropin is recommended compared to what is typically used in women with other indications for $\mathrm{COH}$. We will typically start women with PCOS with 75 units daily of a gonadotropin product with a serum estradiol level planned for the morning of the fourth treatment day. Women must be warned of the risk of cycle cancellation if too many follicles are recruited, the risk of ovarian hyperstimulation (OHSS), and the risk of higher order multiple gestation pregnancy if they choose this mode of treatment. ${ }^{25}$ There are a number of factors that may affect a 
woman's decision whether or not to pursue $\mathrm{COH}$ or skip $\mathrm{COH}$ and move directly to in vitro fertilization. These include the costs of the procedures at an individual center, insurance coverage requirements if she has infertility insurance benefits, and access to centers that provide in vitro fertilization because centers with facilities for IVF are often concentrated in areas of high population density and women may have to travel for hours to get to one of these facilities. ${ }^{40}$ Despite these factors, it may be reasonable for some woman to consider IVF over $\mathrm{COH}$ given the higher success rates associated with IVF and the option for single embryo transfer that exists with lower risks for multiple gestation pregnancy. Given the higher costs associated with IVF, it may be helpful to discuss IVF with women who have PCOS-related infertility early on so they may start to plan ahead should they need to utilize these services.

\section{ART: in vitro fertilization}

IVF offers all women the most effective mode of achieving pregnancy in a single cycle. ${ }^{41}$ On the other hand, IVF may be cost-prohibitive for some women, it is time consuming, and is associated with risks related to OHSS, anesthesia, and surgical complications such as bleeding and bowel/bladder injury. For women with PCOS who have not responded to treatment with more conservative options, or for women with PCOS who have other conditions that require IVF (blocked fallopian tubes, male partner with low sperm counts, or genetic conditions amenable to intervention with preimplantation genetic diagnosis testing) the benefits of IVF may outweigh the risks. For any woman considering IVF treatment, we recommend consulting the SART to obtain information regarding facilities that provide IVF services. ${ }^{42}$ We also recommend investigating the Centers for Disease Control website on ART for additional information regarding factors other than success rates to consider when choosing an IVF facility. ${ }^{43}$ Couples will often consider only one to two cycles of IVF so it is imperative that they are well informed of the success rates in various IVF facilities and the qualifications of their providers.

A recent study found that centers with high-performing IVF programs share common qualities that may be helpful for patients and referring physicians. ${ }^{44}$ One of these commonalities is the selective use of blastocyst embryo transfer which may improve selection for single embryo transfer in IVF. Single embryo transfer may be an especially important option for women with PCOS as they may be at increased risk for pregnancy complications if they were to conceive with multiple gestations given their predisposition for gesta- tional diabetes and preeclampsia. Medical societies like the American Society for Reproductive Medicine and patient advocate groups like RESOLVE are also helpful for couples seeking additional information. ${ }^{45,46}$

Table 1 compares the approximate success rates of various oral ovulation induction agents, $\mathrm{COH}$, and IVF along with their associated side-effects, risks, and relative costs.

\section{Patient treatment preferences for PCOS-related infertility}

Unfortunately, the data are sparse when it comes to incorporating patient preference in the management of PCOSrelated infertility. We found one article when we performed a MEDLINE search for published data using the keywords, "patient preference" and "polycystic ovary syndrome." This article entitled, "Treatment preferences and trade-offs for ovulation induction in CC-resistant patients with polycystic ovary syndrome," assessed the preferences and trade-offs for laparoscopic electrocautery of the ovaries relative to ovulation induction with FSH injections in women with PCOS who were resistant to ovulation induction with $\mathrm{CC} .{ }^{47}$ The data presented in this article suggest that women are open to discussing different treatment options, while weighing the risks and benefits of each.

A discussion of the available options should include a thorough explanation of normal menstrual cycles and ovulation and an explanation of how the various strategies work, their associated risks and benefits, and their cost and efficacy. Unfortunately, inducing ovulation in women with PCOS often requires a trial-and-error process with different medications and lifestyle modifications. The recommendations outlined in this review are consistent with an evidence-based approach to managing PCOS-infertility in a timely, safe, and effective manner. Greater transparency regarding the common difficulties encountered when inducing ovulation in women with PCOS will likely be helpful for improving patient compliance with treatment, although this has not been studied specifically in women with PCOS.

When it comes to patient preference in the management of PCOS-related infertility, the bottom line is that women with infertility ultimately want to conceive. ${ }^{47}$ Personalized treatment algorithms are often dictated by cost, insurance coverage for fertility treatment, and the effectiveness of the various medications in individuals. How these various factors drive a woman's treatment trajectory is yet to be determined, but it is likely that women's choices are often driven by cost and the out-of-pocket-expenses associated with the strategies. In our synthesized data incorporating 


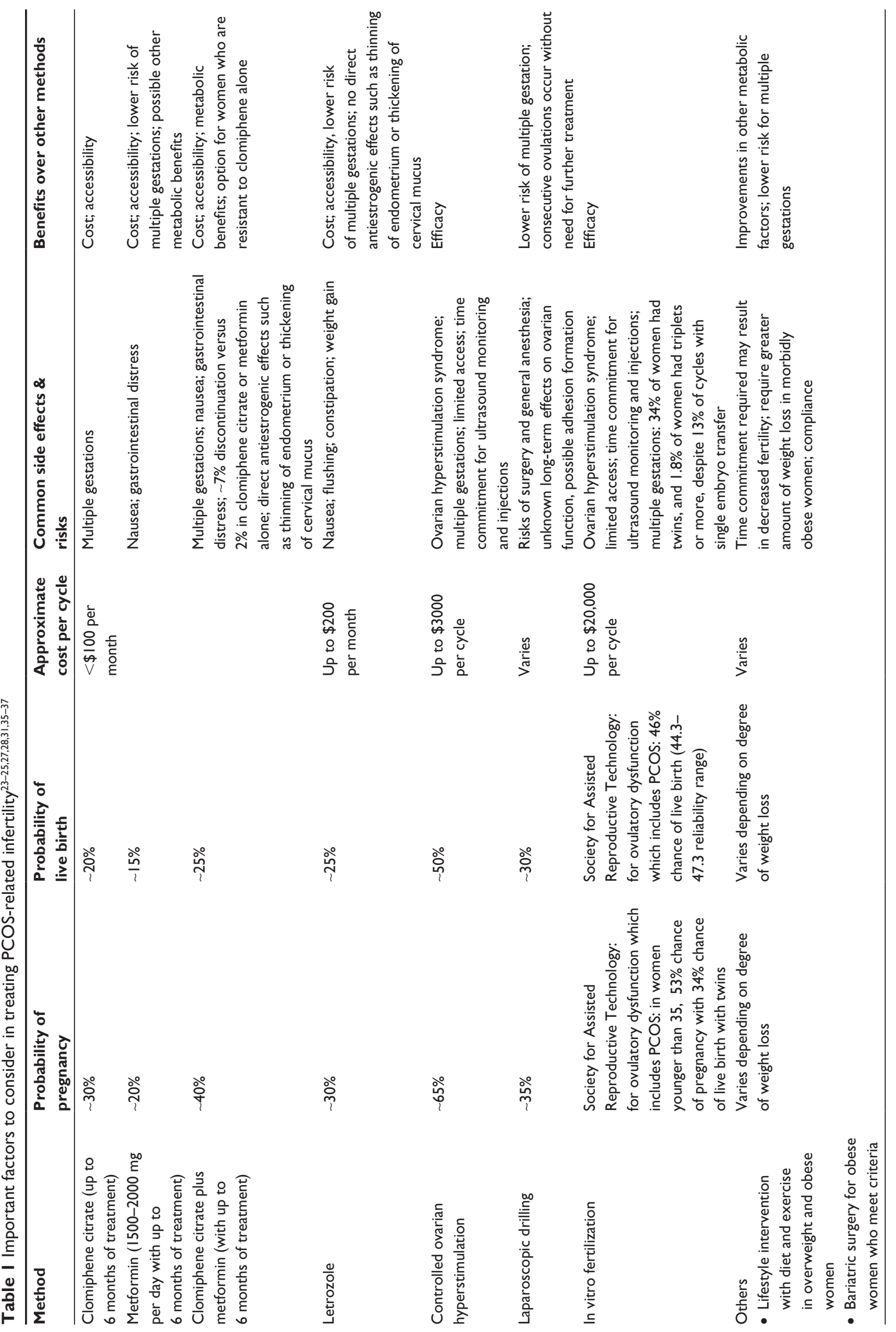


side effects and efficacy into decisions for treating women with PCOS, an approach that combines metformin and CC for initial treatment was the best initial therapy for ovulation induction in women with PCOS, although letrozole was included in the study. Forthcoming data from the NIH-RMN reports will help inform the future use of letrozole in this population.

\section{Keeping women informed, keeping the dialog open, and presenting a global perspective on PCOS-related infertility treatment}

Because of the heterogeneous nature of the presentation of PCOS, it often takes some troubleshooting and trial and error to determine the best course of action. As mentioned above, we recommend screening women with PCOS for related comorbidities as outlined by practice guidelines. ${ }^{6}$ This screening includes checking women for glucose intolerance. If a woman is glucose intolerant, she may benefit from treatment with metformin, but should be warned of side effects and should never be treated with metformin alone if ovulation induction and pregnancy is the goal. If a woman is overweight or obese, lifestyle intervention with the goal of weight loss is also recommended, but is not always rational if a woman has failed numerous attempts at weight loss in the past or if she is older than 35 years. $\mathrm{CC}$ is the proven first-line agent for ovulation induction in women with PCOS, but we are still anticipating the results from the NIH-RMN trial as letrozole may prove to be equally, if not more, efficacious at achieving pregnancy and live birth with reduced risk of multiple gestations. ${ }^{39}$ If these less expensive, easily accessed options prove ineffective, $\mathrm{COH}$ or IVF are possible although they are associated with increased costs, increased time commitment, and increased risks of complications such as ovarian hyperstimulation and multiple gestations.

The good news is infertile women with PCOS have options. It is up to us to ensure that we are fully versed on these options and that we are proactive in educating our patients to ensure that they do not miss the window of opportunity to achieve their family-building goals. If a general practitioner does not have the time to spend counseling and managing women struggling with PCOS-related infertility, a referral to a specialist in reproductive medicine is appreciated by all the parties involved.

\section{Disclosure}

The authors report no conflicts of interest in this work.

\section{References}

1. Stein IF, Leventhal ML. Amenorrhea associated with bilateral polycystic ovaries. Am J Obstet Gynecol. 1935;29:181-191.

2. Zawadski JK, DunaifA. Diagnostic criteria for polycystic ovary syndrome: towards a rational approach. In: Dunaif A, Givens JR, Haseltine FP, Merriam GR, editors. Polycystic Ovary Syndrome. Boston, MA: Blackwell Scientific Publications. 1992:377-384.

3. Azziz R, Carmina E, Dewailly D, et al. The Androgen Excess and PCOS Society criteria for the polycystic ovary syndrome: the complete task force report. Fertil Steril. 2009;91(2):456-488.

4. Fauser BC, Tarlatzis BC, Rebar RW, et al. Consensus on women's health aspects of polycystic ovary syndrome (PCOS): the Amsterdam ESHRE/ASRM-Sponsored 3rd PCOS Consensus Workshop Group. Fertil Steril. 2012;97(1):28-38. e25.

5. Wild RA, Carmina E, Diamanti-Kandarakis E, et al. Assessment of cardiovascular risk and prevention of cardiovascular disease in women with the polycystic ovary syndrome: a consensus statement by the Androgen Excess and Polycystic Ovary Syndrome (AE-PCOS) Society. J Clin Endocrinol Metab. 2010;95(5):2038-2049.

6. ACOG Committee on Practice Bulletins - Gynecology. ACOG Practice Bulletin No 108: Polycystic ovary syndrome. Obstet Gynecol. 2009;114(4):936-949.

7. American Diabetes Association. Standards of medical care in diabetes 2010. Diabetes care. 2010;33(Suppl 1):S11-S61.

8. Sharma ST, Nestler JE. Prevention of diabetes and cardiovascular disease in women with PCOS: treatment with insulin sensitizers. Best Pract Res Clin Endocrinol Metab. 2006;20(2):245-260.

9. Vanky E, Stridsklev S, Heimstad R, et al. Metformin versus placebo from first trimester to delivery in polycystic ovary syndrome: a randomized, controlled multicenter study. J Clin Endocrinol. 2010;95(12): E448-E455.

10. Teitelman M, Grotegut CA, Williams NN, Lewis JD. The impact of bariatric surgery on menstrual patterns. Obes Surg. 2006;16(11): 1457-1463.

11. Sheiner E, Edri A, Balaban E, Levi I, Aricha-Tamir B. Pregnancy outcome of patients who conceive during or after the first year following bariatric surgery. Am J Obstet Gynecol. 2011;204(1):50. e51-e56.

12. Doblado MA, Lewkowksi BM, Odem RR, Jungheim ES. In vitro fertilization after bariatric surgery. Fertil Steril. 2010;94(7): 2812-2814.

13. Luke B, Brown MB, Stern JE, Missmer SA, Fujimoto VY, Leach R; SART Writing Group. Female obesity adversely affects assisted reproductive technology (ART) pregnancy and live birth rates. Hum Reprod. 2011;26(1):245-252.

14. Sneed ML, Uhler ML, Grotjan HE, Rapisarda JJ, Lederer KJ, Beltsos AN. Body mass index: impact on IVF success appears age-related. Hum Reprod. 2008;23(8):1835-1839.

15. Chavarro JE, Rich-Edwards JW, Rosner BA, Willett WC. Diet and lifestyle in the prevention of ovulatory disorder infertility. Obstet Gynecol. 2007;110(5):1050-1058.

16. Chavarro JE, Rich-Edwards JW, Rosner BA, Willett WC. Dietary fatty acid intakes and the risk of ovulatory infertility. Am J Clin Nutr. 2007;85(1):231-237.

17. Chavarro JE, Rich-Edwards JW, Rosner BA, Willett WC. A prospective study of dietary carbohydrate quantity and quality in relation to risk of ovulatory infertility. Eur J Clin Nutr. 2009;63(1):78-86.

18. Chavarro JE, Rich-Edwards JW, Rosner BA, Willett WC. Iron intake and risk of ovulatory infertility. Obstet Gynecol. 2006;108(5): $1145-1152$.

19. Chavarro JE, Rich-Edwards JW, Rosner BA, Willett WC. Use of multivitamins, intake of B vitamins, and risk of ovulatory infertility. Fertil Steril. 2008;89(3):668-676.

20. Balen AH, Anderson RA. Policy and Practice Committee of the BFS. Impact of obesity on female reproductive health: British Fertility Society, Policy and Practice Guidelines. Hum Fertil (Camb). 2007;10(4):195-206. 
21. Bryzyski R, Fox J, Zera C, Lehmann L. Weight limits for access to fertility services: Discriminatory or nonmaleficence? Ken Ryan Ethics Symposium. Proceedings of the 67th Annual Meeting of the American Society for Reproductive Medicine; 2011 Oct 8; Orlando, FO, USA.

22. Jungheim ES, Lanzendorf SE, Odem RR, Moley KH, Chang AS, Ratts VS. Morbid obesity is associated with lower clinical pregnancy rates after in vitro fertilization in women with polycystic ovary syndrome. Fertil Steril. 2009;92(1):256-261.

23. Bayram N, van Wely M, Kaaijk EM, Bossuyt PM, van der Veen F. Using an electrocautery strategy or recombinant follicle stimulating hormone to induce ovulation in polycystic ovary syndrome: randomised controlled trial. BMJ. 2004;328(7433):192.

24. Farquhar CM, Williamson K, Gudex G, Johnson NP, Garland J Sadler L. A randomized controlled trial of laparoscopic ovarian diathermy versus gonadotropin therapy for women with clomiphene citrate-resistant polycystic ovary syndrome. Fertility and Sterility. 2002;78(2):404-411

25. Farquhar C, Lilford RJ, Marjoribanks J, Vandekerckhove P. Laparoscopic 'drilling' by diathermy or laser for ovulation induction in anovulatory polycystic ovary syndrome. Cochrane Database Syst Rev. 2007;(3): CD001122.

26. Nestler JE, Jakubowicz DJ, Evans WS, Pasquali R. Effects of metformin on spontaneous and clomiphene-induced ovulation in the polycystic ovary syndrome. N Engl J Med. 1998;338(26):1876-1880.

27. Palomba S, Pasquali R, Orio F Jr, Nestler JE. Clomiphene citrate, metformin or both as first-step approach in treating anovulatory infertility in patients with polycystic ovary syndrome (PCOS): a systematic review of head-to-head randomized controlled studies and meta-analysis. Clinical Endocrinology. 2009;70(2):311-321.

28. Moll E, Bossuyt PM, Korevaar JC, Lambalk CB, van der Veen F. Effect of clomifene citrate plus metformin and clomifene citrate plus placebo on induction of ovulation in women with newly diagnosed polycystic ovary syndrome: randomised double blind clinical trial. BMJ. 2006;332(7556):1485

29. Legro RS, Barnhart HX, Schlaff WD, et al. Clomiphene, metformin, or both for infertility in the polycystic ovary syndrome. $N$ Engl J Med. 2007;356(6):551-566.

30. Geberhiwot T, Jones AF. Treatment of infertility in the polycystic ovary syndrome. $N$ Engl J Med. 2007;356(19):2000; author reply 1999-2001.

31. McGovern PG, Carson SA, Barnhart HX, et al. Medication adherence and treatment success in the National Institute of Child Health and Human Development-Reproductive Medicine Network's Pregnancy in Polycystic Ovary Syndrome Trial. Fertil Steril. 2008;90(4):1283-1286.

32. Jungheim ES, Odibo AO. Fertility treatment in women with polycystic ovary syndrome: a decision analysis of different oral ovulation induction agents. Fertil Steril. 2010;94(7):2659-2664.

33. Myers ER, Silva SG, Hafley G, Kunselman AR, Nestler JE, Legro RS Estimating live birth rates after ovulation induction in polycystic ovary syndrome: sample size calculations for the pregnancy in polycystic ovary syndrome trial. Contemp Clin Trials. 2005;26(3):271-280.
34. Legro RS. Metformin during pregnancy in polycystic ovary syndrome: another vitamin bites the dust. J Clin Endocrinol Metab. 2010;95(12):5199-5202.

35. Mitwally MF, Casper RF. Use of an aromatase inhibitor for induction of ovulation in patients with an inadequate response to clomiphene citrate. Fertil Steril. 2001;75(2):305-309.

36. Atay V, Cam C, Muhcu M, Cam M, Karateke A. Comparison of letrozole and clomiphene citrate in women with polycystic ovaries undergoing ovarian stimulation. J Int Med Res. 2006;34(1):73-76.

37. He D, Jiang F. Meta-analysis of letrozole versus clomiphene citrate in polycystic ovary syndrome. Reprod Biomed Online. 2011;23(1):91-96.

38. Zeinalzadeh M, Basirat Z, Esmailpour M. Efficacy of letrozole in ovulation induction compared to that of clomiphene citrate in patients with polycystic ovarian syndrome. J Reprod Med. 2010;55(1-2):36-40.

39. Legro RS, Kunselman AR, Brzyski RG, et al; for the NICHD Reproductive Medicine Network. The Pregnancy in Polycystic Ovary Syndrome II (PPCOS II) trial: Rationale and design of a double-blind randomized trial of clomiphene citrate and letrozole for the treatment of infertility in women with polycystic ovary syndrome. Contemp Clin Trials. 2012;33(3):470-481.

40. Nangia AK, Likosky DS, Wang D. Distribution of male infertility specialists in relation to the male population and assisted reproductive technology centers in the United States. Fertil Steril. 2010;94(2):599-609.

41. Van Voorhis BJ. Clinical practice. In vitro fertilization. $N$ Engl J Med. 2007;356(4):379-386.

42. sart.org [homepage on the Internet]. SART, Society for Assisted Reproductive Technology; 1996-2012. Available from: http://sart.org/. Accessed 2 February, 2012.

43. CDC.gov [homepage on the Internet]. Annual ART success rates reports. Atlanta, GA: Centers for Disease Control and Prevention; 2012. Available from: http://www.cdc.gov/art/ ARTReports.htm. Accessed 2 February, 2012.

44. Van Voorhis BJ, Thomas M, Surrey ES, Sparks A. What do consistently high-performing in vitro fertilization programs in the US do? Fertil Steril. 2010;94(4):1346-1349.

45. ASRM.org [homepage on the Internet]. ASRM, American Society for Reproductive Medicine, 1996-2012. Available from: http://asrm.org/ ASRM_homepage/. Accessed 2 February, 2012.

46. RESOLVE.org [homepage on the Internet]. McLean, VA: RESOLVE: The National Infertility Association; 2012. Available from: http://www. resolve.org/. Accessed 2 February, 2012.

47. Bayram N, van Wely M, van der Veen F, Bossuyt PM, Nieuwkerk P. Treatment preferences and trade-offs for ovulation induction in clomiphene citrate-resistant patients with polycystic ovary syndrome. Fertil Steril. 2005;84(2):420-425.
Patient Preference and Adherence

\section{Publish your work in this journal}

Patient Preference and Adherence is an international, peer-reviewed, open access journal focusing on the growing importance of patient preference and adherence throughout the therapeutic continuum. Patient satisfaction, acceptability, quality of life, compliance, persistence and their role in developing new therapeutic modalities and compounds to

\section{Dovepress}

optimize clinical outcomes for existing disease states are major areas of interest. This journal has been accepted for indexing on PubMed Central. The manuscript management system is completely online and includes a very quick and fair peer-review system. Visit http://www.dovepress.com/ testimonials.php to read real quotes from published authors. 